\title{
Analytical Model of Energy Consumption in Cluster based Wireless Sensor Networks with Data Aggregation using M/M/1 Queuing Model
}

\author{
Manijeh \\ Keshtgary \\ Department of \\ Computer \\ Engineering \& IT \\ Shiraz University of \\ Technology, Iran \\ Shiraz, Iran
}

\author{
Mohammad Reza \\ Mansouri \\ Department of \\ Computer \\ Engineering \& IT \\ Shiraz University of \\ Technology, Iran \\ Shiraz, Iran
}

\author{
Reza \\ Mohammadi \\ Department of \\ Computer \\ Engineering and \\ Information \\ Technology \\ Shiraz University of \\ Technology \\ Shiraz, Iran
}

\author{
Mohammad \\ Mahmoudi \\ Department of \\ Computer \\ Engineering \& IT \\ Shiraz University of \\ Technology, Iran \\ Shiraz, Iran
}

\begin{abstract}
Wireless Sensor Networks (WSNs) consist of several sensor nodes equipped with limited power sources that sense environments event and transmit data to the sink node. WSNs have many constraints including energy, redundant data, and many-to-one flows. Data aggregation is one of the most important issues for achieving energy-efficiency in wireless sensor networks. Sensor nodes in the surrounding region of an event may generate redundant sensed data. A data aggregation technique in WSNs focuses on decreasing the energy consumption by reducing the amount of data that needed to be sent to the sink node. In this paper, we propose an M/M/1 queuing model for evaluating energy consumption in WSNs with dada transmission. The proposed model can help designers to evaluate the energy consumption of cluster based wireless sensor network with data aggregation. We compare the energy consumption results for networks with and without data aggregation technique.
\end{abstract}

\section{General Terms}

Energy consumption in cluster based wireless sensor networks with data aggregation.

\section{Keywords}

Wireless sensor networks, data aggregation, M/M/1 queuing model, energy consumption.

\section{INTRODUCTION}

WSNs consist of large number of distributed sensor nodes with the ability to monitor physical or environmental conditions, such as temperature, vibration, sound, pressure, humidity or motion. Sensor nodes pass their data through the network to a main location, external sink or a base-station. A sensor node generates data and transmit sensed data packet to the base station that could be connected to the internet. WSNs can be used for many applications including military supervision, habitat, indoor and environmental monitoring [1]-[3]. However WSNs have many challenges in use and deployment including limited power resources and also limited memory and communication capabilities [4].

However, the most important challenges in the WSNs are energy consumption because battery capacities of sensor nodes are limited and replacing them in many applications are impractical. Sensor nodes consume most energy for data transmission and reception [5]; therefore reducing amount of transmitted data can increase network life time and its mission ability. In order to reduce data transmission rate, sensor node's data can be aggregated and then sent to the base station. The goal of data aggregation is the elimination of redundant data transmission by summarization sensed data.

Many data aggregation techniques and protocols are proposed in recent years. However, the architecture of the WSNs plays a crucial role in the performance of data aggregation protocols and techniques. In this paper, we propose an analytical model for evaluating energy consumption in term of data transmission, reception and aggregation in cluster based WSN architecture using $\mathrm{M} / \mathrm{M} / 1$ queuing model.

The rest of this paper is organized as follows. In section 2, we present the data aggregation principle and goals. In section 3 , we present our proposed energy consumption model. In section 4 , we compare energy consumption in the network with and without data aggregation technique. We conclude our paper in section 5 .

\section{DATA AGGREGATION}

In most WSNs, sensor nodes monitor environment events and transmit sensed data to the base station. WSNs usually consist of large number of sensor nodes that densely deployed; this means close sensor nodes could sense the same event as other nodes and we have similar and redundant data. Therefore transmitting all the data is unnecessary. Data transmitted in WSNs is different from traditional wireless networks. In WSNs, end-user analyzes the collected data and determines some event about the monitored environment [6].

In order to save resources and reduce consuming energy for the data transmission, sensor nodes data can be processed, eliminate the redundant data and send the result to the base station. For instance, if sensors are monitoring humidity in precision agriculture application, the humidity at different points of a domain correlated and the end users are only need to determine the average humidity in the domain. So sensors data in each domain can be aggregated and the result would be send to the base station. Using this technique will minimize the transmission rate and also save energy and increase network lifetime [7].

The type of data aggregation technique and protocol that needs to be performed depends on the application, user requirements and network architecture. There has been extensive work on data aggregation technique in WSNs. 
Selection of proper data aggregation technique depends on How data is gathered at the sensor nodes and also how packets are routed through the network [8], [9].

The performance of different data aggregation protocols and technique depend on architecture of the WSNs. We can divide WSNs architecture in two category; Flat and Hierarchical networks. In flat networks, role and equipment of each sensor node are equal and so the same battery power exist in each sensor node. In such networks, data aggregation is complete by use of data centric routing protocol. For example base station transmits a query message to the network and sensor nodes which have data matching the query send response messages back to the sink. For this category there is some communication protocol such as Push diffusion, Directed. In hierarchical network, data fusion is done at the special nodes, which reduces the number of messages transmitted to the base station. We can divide hierarchical network in four categories; Cluster-Based Network, ChainBased network, Tree-Based and Grid-Based network. For each category one or more data aggregation protocol proposed. For example in Cluster-Based Networks; LowEnergy Adaptive Clustering Hierarchy (LEACH), Hybrid Energy Efficient Distributed Clustering Approach (HEED) and clustered diffusion with dynamic data aggregation (CLUDDA).For more information about aggregation protocol based on WSNs architecture referee to [10]-[12]. In this paper we focus on Cluster-Based Network architecture.

In most WSNs with energy limitation, it is inefficient for sensor nodes to transmit the data directly to the base station. Sensor nodes can transmit data to a local sink node or cluster head which aggregates data from all the sensor nodes in its cluster and transmits the result to the base station. Clustering is the process of grouping sensor nodes in a densely and large-scale WSNs. The process of clustering in a WSNs involved with some issue; such as how many clusters should be formed, how many nodes can be taken in to a single cluster and how select the cluster-head node in each cluster. However we can choose some powerful nodes, in terms of energy or processing capacity, as a cluster-head node.

For this manner we can divide WSNs nodes in three categories based on that's operation and responsibility. Simple regular sensor nodes that known as cluster-member (CM), cluster-head $(\mathrm{CH})$ node and base station (BS). CM sense environment properties periodically or only sense event and send related data to the $\mathrm{CH}$ node that basically collect data from multiple $\mathrm{CM}$ in its cluster, using some aggregation function like sum, count, average, max or min aggregates collected data packet and then sends aggregated result to BS. Figure 1 shows this process.

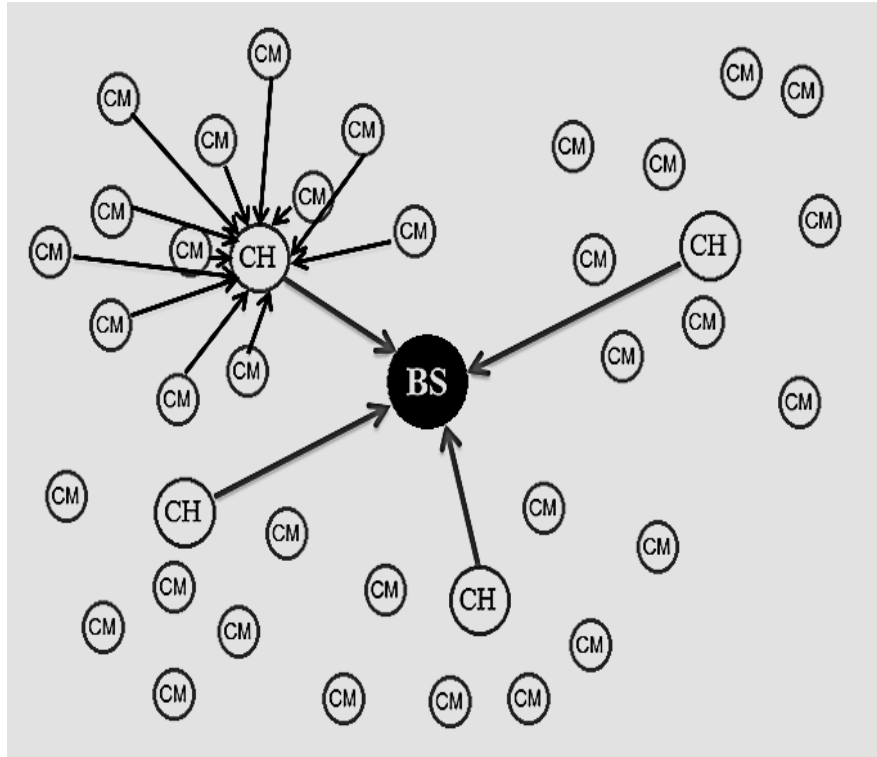

Fig 1: cluster-based data aggregation

\section{ENERGY CONSUMPTION MODEL}

In this section, we propose an analytical model based on $\mathrm{M} / \mathrm{M} / 1$ queue for energy consumption. Sensor nodes consume their energy for three basic operations. Firs in acquisition operation that sensor node convert the sensed event to a digital signal. Second in processing operation that digital signal may be processed before transmission and finally for transmission or reception of data to or from another sensor node. In this work, we focus on the energy consumption for transmitting or receiving and also processing the data packets. We assume that the nodes could be in two modes, sleep and active mode. In sleep mode, the node is in sleep mode and wake up in the next interval. In active mode, node can transmit or receive data and also can process data using data aggregation techniques. Note that sensor node cannot send and receive data at the same time because of half duplex radio channel.

In our model, we assume that $\mathrm{CM}$ nodes periodically sense environment and send sensed data to the $\mathrm{CH}$ node. $\mathrm{CH}$ node after reception of data will aggregate data and transmits the result to the base station. To compute energy consumed for transmitting data to the base station, we model each node based on simple $\mathrm{M} / \mathrm{M} / 1$ queue. In this manner, service time in transmitter or receiver queue equals to the time spent for transmission or reception of a data packet. Therefore we consider each data packet as a job in the system. Figure 2 shows system as a network of queue. 


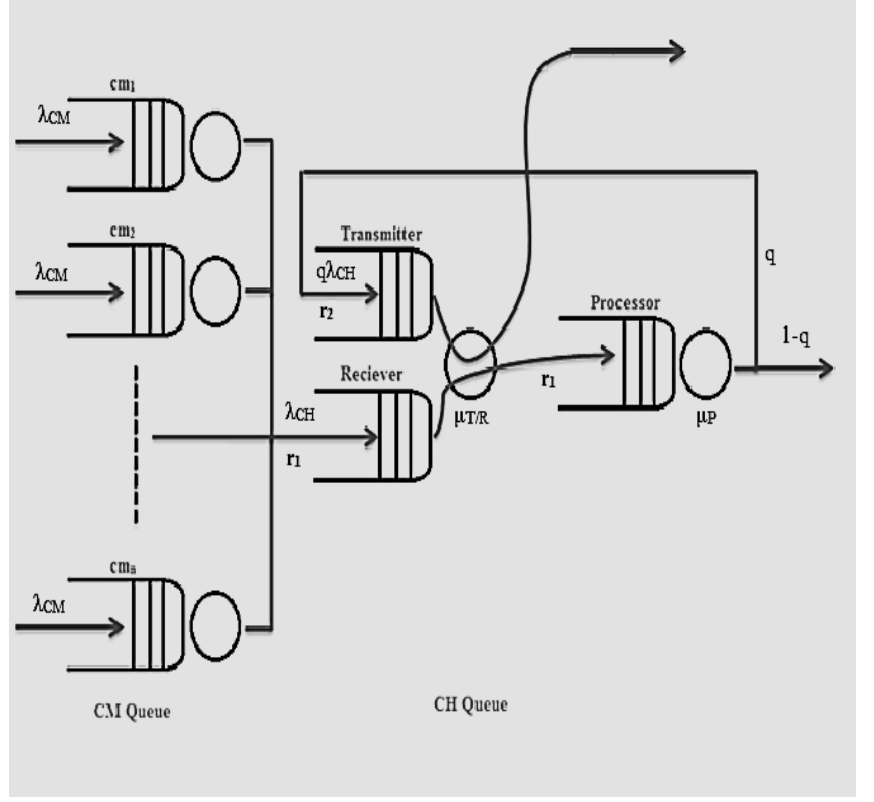

Fig 2: system as a queuing network

\subsection{Model assumptions}

For analyzing model we made following assumptions:

- $\quad$ All CM nodes in network are identical.

- $\quad$ All $\mathrm{CH}$ nodes in network are identical.

- The arrival of data packets to CM's transmitter queue is assumed to follow a Poisson process with mean arrival rate $\lambda_{\mathrm{CM}}$. Therefore the arrival of data packets to CH's transmitter queue is $\lambda_{\mathrm{CH}}=(\mathrm{N}-1) *$ $\lambda_{\mathrm{CM}}$ if each cluster consists of $\mathrm{N}$ node.

- Data packets after and before aggregation are of fixed size.

- $\quad$ Each CM nodes sends packets to $\mathrm{CH}$ independently and identically and the service rates following exponential distribution with parameter $\mu_{\mathrm{T} / \mathrm{R}}$ for transceiver queue and $\mu_{\mathrm{p}}$ for $\mathrm{CH}$ processor queue.

- Numbers of CM nodes in each cluster are identical.

- For each queue $\lambda<\mu$.

In our analytical model, we define following notations:

- $\quad \mathrm{R}$ : the number of job classes in the network.

- $\mu_{\mathrm{i}, \mathrm{r}}$ : the service rate of the $\mathrm{i}^{\text {th }}$ station of the $\mathrm{r}^{\text {th }}$ class.

- $\mathrm{P}_{\mathrm{i} . \mathrm{r} ; \mathrm{j}, \mathrm{s}}$ : probability that a job of the $\mathrm{r}^{\text {th }}$ class at the $\mathrm{i}^{\text {th }}$ station is transferred to the $\mathrm{s}^{\text {th }}$ class and the $j^{\text {th }}$ station (routing probability).

- $\mathrm{P}_{0 ; \mathrm{j}, \mathrm{s}}$ : in an open network the probability that a job from outside the network enters to the $\mathrm{j}^{\text {th }}$ station of the $\mathrm{s}^{\text {th }}$ class.

- $\mathrm{P}_{\mathrm{i}, \mathrm{r} ; 0}$ : in an open network the probability that a job of the $r^{\text {th }}$ class leaves the network after having been served at the $\mathrm{i}^{\text {th }}$ station.
- $\quad \rho_{\mathrm{i}}$ : utilization of the $\mathrm{i}^{\text {th }}$ station for all classes of jobs.

- $\quad P_{i}(0)$ : probability that the $i^{\text {th }}$ station be empty.

- $\mathrm{E}_{\text {sleep: }}$ energy consumption in sleep state.

- $\quad \mathrm{E}_{\text {idle: }}$ energy consumption in idle state.

- $\quad \mathrm{N}$ : Number of nodes in each cluster.

\subsection{Energy consumption in $\mathrm{CM}$ nodes}

First we model energy consumption in $\mathrm{CM}$ nodes. Based on the $M / M / 1$ queuing model, the equilibrium state probabilities of $n$ jobs in the CM's transceiver are given by [13]:

$$
\begin{aligned}
& P_{C M}(n)=\left(1-\rho_{C M}\right) * \rho_{C M}^{n} \\
& \text { that } \rho_{C M}=\frac{\lambda_{C M}}{\mu_{T / R}}
\end{aligned}
$$

Where $\lambda_{\text {СM }}$ is the mean arrival rate of CM's transmitter queue and $\mu_{\mathrm{T} / \mathrm{R}}$ is the mean service rate in $\mathrm{CM}$ and $\mathrm{CH}$ transmitter or receiver queues that:

$$
\mu_{T / R}=\frac{\text { Band Width }}{\text { Packet Size }}
$$

So energy consumption in each CM nodes for transmitting data to $\mathrm{CH}$ is:

$$
\begin{aligned}
& E C_{C M}=\left(P_{C M}(0) * E_{\text {sleep }}\right)+[(1- \\
& \left.\left.P_{C M}(0)\right) *\left(E_{\text {idle }}+E_{T / C M}\right)\right](3)
\end{aligned}
$$

That based on (1):

$$
P_{C M}(0)=1-\rho_{C M}
$$

where in (3) $\mathrm{EC}_{\mathrm{CM}}$ is the total energy consumed in each $\mathrm{CM}$ for transmitting data in given time interval and $\mathrm{E}_{\mathrm{T} / \mathrm{CM}}$ is the transmission energy in $\mathrm{CM}$ nodes.

\subsection{Energy consumption in $\mathrm{CH}$ nodes}

For modeling energy consumption in $\mathrm{CH}$ nodes, we consider $\mathrm{CH}$ nodes as an open BCMP queuing network model that consists of 2 service stations and 2 job classes. A queuing network may be open (Jackson model), closed (Gordon/Newell model), or mixed that just consist of single classes of job. Jackson and Gordon/Newell model were extended by Baskett, Chandy, Muntz, and Palacios that consider multiple classes of job in the network [14], [15]. Our queuing network consists of 2 service stations that each service station contains a single-server with first-come-firstserved (FCFS) policy and equal service rate for all classes of jobs. This feature causes our server station become type one based on Baskett server type category. In this type of station, the service times must be exponentially distributed and classindependent. Therefore for $\mathrm{i}^{\text {th }}$ service station, $\mu_{\mathrm{i} 1}=\mu_{\mathrm{i} 2}=\ldots=\mu_{\mathrm{iR}}=\mu_{\mathrm{i}}$. We assume that the number of jobs (data packet) in each class at each service station (processor and transmitter/receiver) is always non negative. For analyzing transceiver in term of transmission after aggregation, we define 2 different classes of jobs. We assume that jobs change their classes while transferred through the processor station and transceiver station after data aggregation. In our BCMP model, there are 2 stations and 2 job classes. For each class, we specify routing probabilities through the network in Table 
1. Jobs are transferred between any two stations and may be changing its classes according to given routing probabilities. For example (see Table 1), a class $\mathrm{r}_{1}$ job entering from outside goes to the transmitter/receiver queue (T/R) with probability $\mathrm{P}_{0 ; \mathrm{T} / \mathrm{R}, \mathrm{r} 1}=1$.

Table 1: routing probabilities

\begin{tabular}{|c|c|c|c|c|c|}
\hline$\overline{\mathbf{P}_{\mathrm{i} . \mathrm{r} ; \mathrm{j}, \mathrm{s}}}$ & 0 & $\mathbf{T} / \mathbf{R}, \mathbf{r}_{1}$ & $\mathbf{T} / \mathbf{R}, \mathbf{r}_{2}$ & $\mathbf{P}, \mathbf{r}_{1}$ & $\mathbf{P}, \mathbf{r}_{2}$ \\
\hline 0 & 0 & 1 & 0 & 0 & 0 \\
\hline $\mathbf{T} / \mathbf{R}, \mathbf{r}_{1}$ & 0 & 0 & 0 & 1 & 0 \\
\hline $\mathbf{T} / \mathbf{R}, \mathbf{r}_{2}$ & 1 & 0 & 0 & 0 & 0 \\
\hline $\mathbf{P}, \mathbf{r}_{1}$ & $1-q$ & 0 & $q$ & 0 & 0 \\
\hline $\mathbf{P}, \mathbf{r}_{2}$ & 0 & 0 & 0 & 0 & 0 \\
\hline
\end{tabular}

With that assumption and routing probabilities table, we can set the arrival rate of each class to each node using the following formula:

$e_{i, r}=\lambda_{0 ; i, r}+\sum_{j=1 . . N}^{s \in R} \mathrm{e}_{j, s} P_{j, s ; i, r}$

where $\lambda_{0: \text { ir }}$ is the arrival rate from outside to the $\mathrm{i}^{\text {th }}$ station of the $\mathrm{r}^{\text {th }}$ class and $\mathrm{e}_{\mathrm{i}, \mathrm{r}}$ is the visit rate of jobs of the $\mathrm{r}^{\text {th }}$ class at the $\mathrm{i}^{\text {th }}$ station.

so utilization of each class equals to:

$\rho_{i, r}=\frac{e_{i, r}}{\mu_{i}}$

where $\rho_{\mathrm{i}, \mathrm{r}}$ is utilization of the $\mathrm{i}^{\text {th }}$ station of the $\mathrm{r}^{\text {th }}$ class.

Therefore for each station and its class:

$\rho_{T / R, r 1}=\frac{\lambda_{C H}}{\mu_{T / R}}, \rho_{T / R, r 2}=\frac{q \lambda_{C H}}{\mu_{T / R}}, \rho_{P, r 1}=\frac{\lambda_{C H}}{\mu_{P}}$

where $\lambda_{\mathrm{CH}}$ is the mean arrival rate of $\mathrm{CH}$ 's transmitter queue, $\mu_{\mathrm{p}}$ is the mean service rate in $\mathrm{CH}$ processor queue that $\mu_{P}=\frac{1}{\text { Packet Size } * \text { one bit processing time }}$

and 1-q is the probability that a job after aggregation, eliminated in processor station.

For simplification, we model the total number of jobs in each service station in equilibrium state probabilities function instead of the number of each class of jobs in each service station. For the open queuing network [15], we will have the following equation:

$$
\begin{aligned}
& P_{C H}(S)=P\left(n_{1}, n_{2}\right)=\left(1-\lambda_{C H} \rho_{T / R}\right) * \\
& \left(1-\lambda_{C H} \rho_{P}\right) * \lambda_{C H}{ }^{n_{1}+n_{2}} * \rho_{T / R}{ }^{n_{1}} * \rho_{T / R}{ }^{n_{1}}
\end{aligned}
$$

where:

$$
\rho_{T / R}=\frac{\lambda_{C H}(q+1)}{\mu_{T / R}}
$$

$\rho_{P}=\frac{\lambda_{C H}}{\mu_{P}}$

Now we can model the energy consumption in $\mathrm{CH}$ node:

$$
\begin{aligned}
& E C_{C H}=\left(P_{C H}(0) * E_{\text {sleep }}\right)+[(1- \\
& \left.P_{C M}(0) * E_{\text {idle }}\right]+\left(E T_{C H}+E P_{C H}+E R_{C H}\right)
\end{aligned}
$$

where $\mathrm{EC}_{\mathrm{CH}}$ is the total energy consumed in each $\mathrm{CH}$ for receiving, processing and transmitting data in given time interval, $\mathrm{ET}_{\mathrm{CH}}$ is the total energy consumed in each $\mathrm{CH}$ for transmitting data in given time interval, $\mathrm{ER}_{\mathrm{CH}}$ is the total energy consumed in each $\mathrm{CH}$ for receiving data in given time interval and $\mathrm{EP}_{\mathrm{CH}}$ is the total energy consumed in each $\mathrm{CH}$ for processing data in given time interval.

where in (8):

$$
\begin{aligned}
& P_{C H}(0)=P(0,0)=\left(1-\lambda_{C H} \rho_{T / R}\right) *(1- \\
& \left.\lambda_{C H} \rho_{P}\right) \\
& E T_{C H}=\rho_{T / R, r 2} * E_{T / C H} \\
& E R_{C H}=\rho_{T / R, r 1} * E_{R / C H} \\
& E P_{C H}=\rho_{P, r 1} * E_{P / C H}
\end{aligned}
$$

Where $\mathrm{E}_{\mathrm{T} / \mathrm{CH}}$ is the transmission energy in $\mathrm{CH}$ nodes, $\mathrm{E}_{\mathrm{R} / \mathrm{CH}}$ is the receiving energy in $\mathrm{CH}$ nodes and $\mathrm{E}_{\mathrm{P} / \mathrm{CH}}$ is the processing energy in $\mathrm{CH}$ nodes, and

$$
E_{P / C H}=\frac{\text { energy consumed for processing one bit }}{\text { one bit processing time }} .
$$

So total energy consumed in each cluster equals to the following equation:

$$
E T=E C_{C H}+(N-1) * E C_{C M}
$$

Where ET is the total energy consumed in each cluster in given time interval.

\section{MODEL ANALYSIS}

In this section, we compare the result of proposed model with result of model that created for WSNs without data aggregation technique. We use the proposed analytical models in WSNs using the parameters given in Table 2. Model results are obtained in various scenarios by varying the mean arrival rate per $\mathrm{CM}$ and varying the elimination probability after data aggregation in $\mathrm{CH}$ to determine the average energy consumption. 
Table 2: Model and simulation parameter

\begin{tabular}{|c|c|}
\hline Mean arrival rate to CM'stransmitterqueue & 0.1 to 1 \\
\hline Packet size & $200 \mathrm{~B}$ \\
\hline Number of node in each cluster & 10 \\
\hline Band Width & $350 \mathrm{Kbps}$ \\
\hline Elimination probability(1-q) & 0.1 to 0.9 \\
\hline One bit processing time & $30 \mathrm{~ns}$ \\
\hline $\mathrm{E}_{\text {sleep }}$ & $75 \mu \mathrm{j}$ \\
\hline $\mathrm{E}_{\mathrm{idle}}$ & $6 \mathrm{mj}$ \\
\hline One bit processing energy consumption & $25 \mathrm{nj}$ \\
\hline $\mathrm{E}_{\mathrm{T} / \mathrm{CM}}$ & $30 \mathrm{mj}$ \\
\hline $\mathrm{E}_{\mathrm{T} / \mathrm{CH}}$ & $36 \mathrm{mj}$ \\
\hline $\mathrm{E}_{\mathrm{R} / \mathrm{CH}}$ & $12 \mathrm{mj}$ \\
\hline Time & $1000 \mathrm{~s}$ \\
\hline
\end{tabular}

For achieving result of energy consumption in WSNs without data aggregation, we use (13) and (14) for $\mathrm{EC}_{\mathrm{CH}}$ :

$$
\begin{gathered}
E C_{C H}=\left(P_{C H}(0) * E_{\text {sleep }}\right)+[(1- \\
\left.P_{C M}(0) * E_{\text {idle }}\right]+\left(E T_{C H}+E R_{C H}\right)
\end{gathered}
$$

that:

$P_{C H}(0)=\left(1-2 \lambda_{C H} \rho_{T / R}\right)$

$$
\begin{aligned}
& E T_{C H}=\rho_{T / R, r 1} * E_{T / C H} \\
& E R_{C H}=\rho_{T / R, r 1} * E_{R / C H}
\end{aligned}
$$

and:

$\rho_{T / R, r 1}=\frac{\lambda_{C H}}{\mu_{T / R}}, \rho_{T / R, r 2}=\frac{\lambda_{C H}}{\mu_{T / R}}$

For calculating energy consumption in CM nodes we used (3).

In Figure 3, we can see the total energy consumption in one cluster by using analytical model with data aggregation and analytical model without data aggregation. In each figure, we compute energy consumption with different mean arrival rate to CM's transmitter queue. As we can see, increasing the mean arrival rate would result in increasing energy consumption. We consider different elimination probability. We can see that for elimination probability less than 0.6, energy consumption for network with data aggregation is greater than network without data aggregation. However this result depends on the value of WSN parameters that illustrated in table 2.

\section{CONCLUSIONS}

In this paper we have proposed the new simple energy consumption estimation scheme in cluster based WSN with data aggregation using queuing network model. The main goal of data aggregation is decreasing energy consumption by decreasing need for redundant data transmission. For comparison of network with data aggregation and network without it, we proposed another analytical model. After comparison result of two models in various scenarios, we can see that energy consumption in best state $25 \%$ decreased, also for elimination probability less than 0.6 , energy consumption for network with data aggregation is greater than network without data aggregation. However those values are based on ours case study and may be different in other case study with various WSNs parameters.

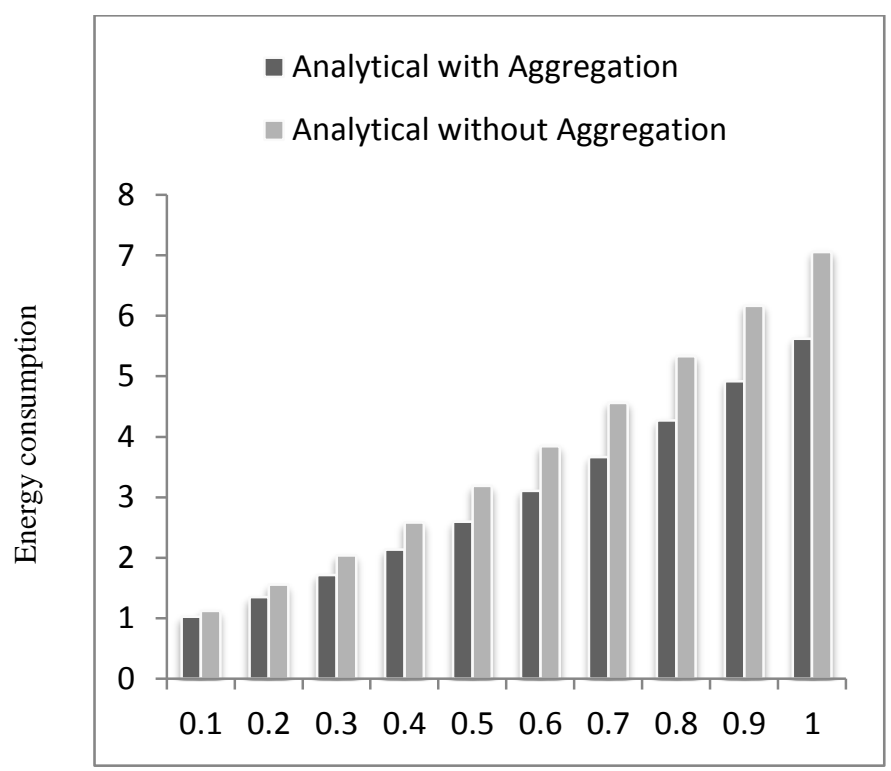

Arrival rate

3-a

\section{- Analytical with Aggregation \\ - Analytical without Aggregation}

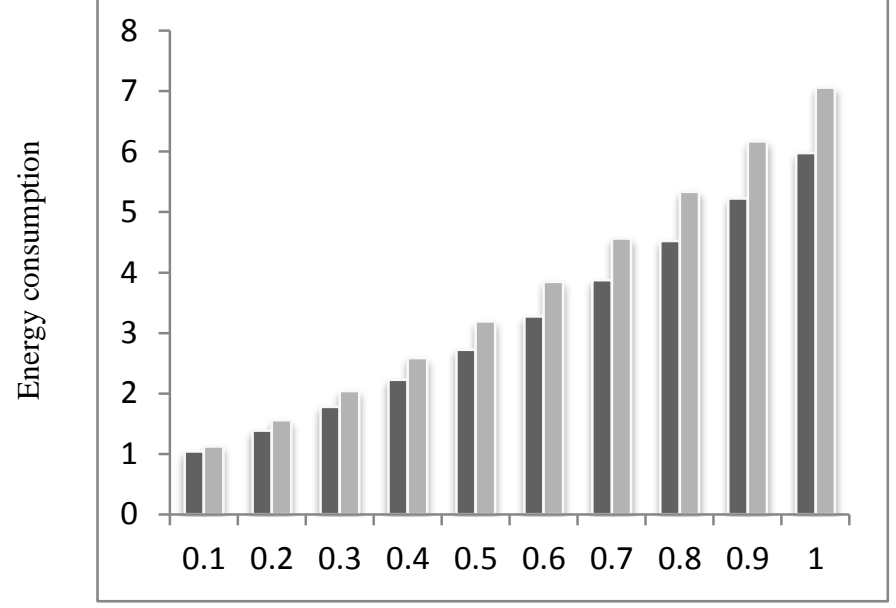

Arrival rate

3-b 

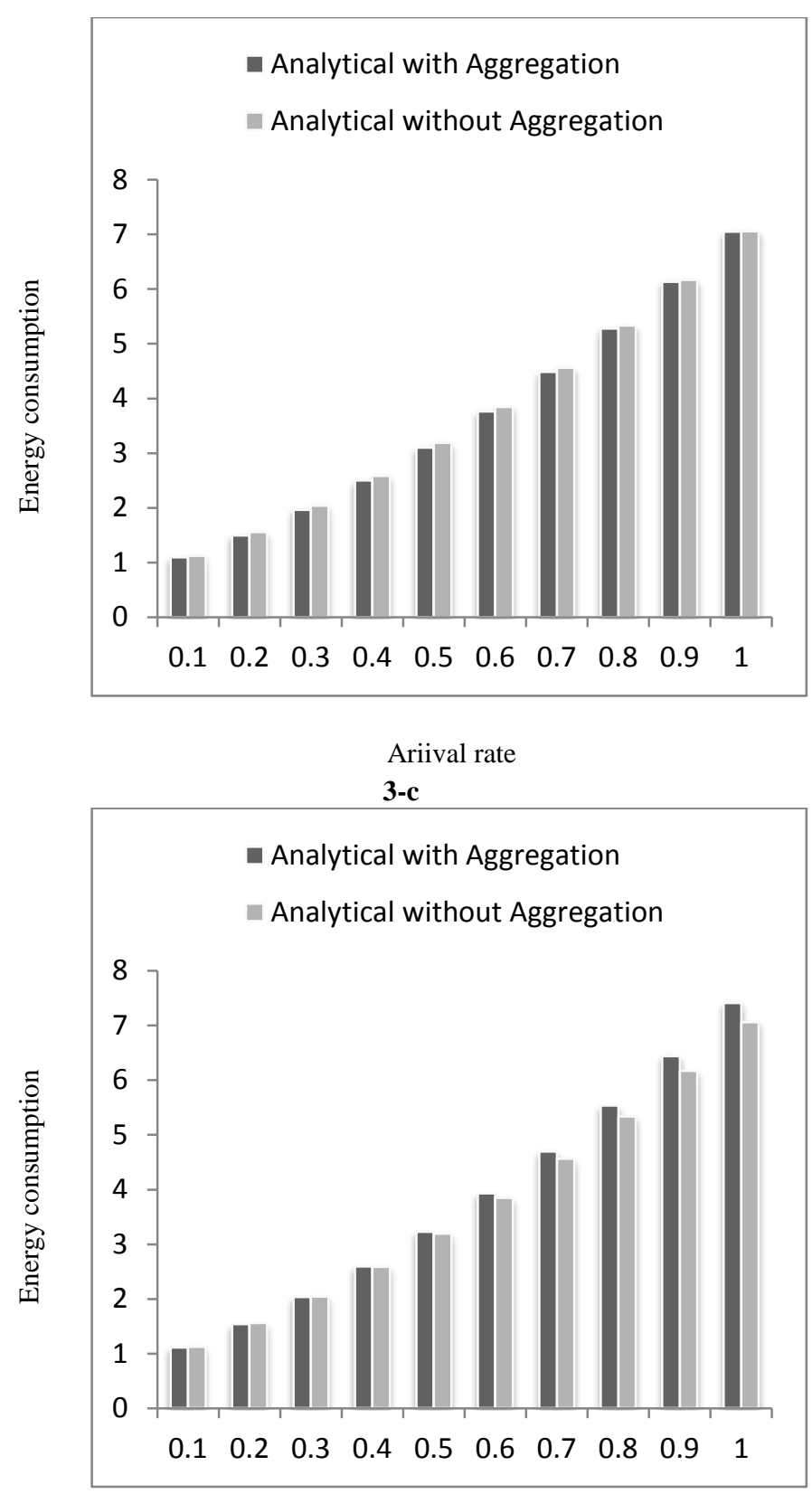

$$
\begin{aligned}
& \text { Arrival rate } \\
& \text { 3-d }
\end{aligned}
$$

Fig 3: energy consumption for $\mathrm{a}: \mathrm{q}=\mathbf{0 . 1}, \mathrm{b}: \mathrm{q}=\mathbf{0 . 2}, \mathrm{c}: \mathrm{q}=\mathbf{0 . 5}$, $\mathrm{d}: \mathrm{q}=\mathbf{0 . 6}$

\section{REFERENCES}

[1] C. Shen, C. Srisathapornphat, and C. Jaikaeo, "Sensor information networking architecture and applications," IEEE Personnel Communications, Aug. 2001, pp.52-59.

[2] S. Tilak, N. Abhu-Gazhaleh, and W. R. Heinzelman, "A taxanomy of wireless micro-sensor network models," ACM SIGMOBILE Mobile Comp. Commun. Rev. , vol. 6, no. 2, Apr. 2002, pp. 28-36.
[3] A. Cerpa et al., "Habitat monitoring: Application driver for wireless communications technology," 2001 ACM SIG-COMMWorkshop on Data Communications in Latin America and the Caribbean , Costa Rica, April 2001.

[4] I. Akyldiz, W.Su, Y. Sankarasubramanian and E. Cayirci, "A survey on sensor networks," IEEE Commun. Mag., vol. 40, no. 8, Aug. 2002, pp. 102-14.

[5] R.B. Patel, D. Kumar, and T.C Aseri, "EECDA: Energyefficient Clustering and Data Aggregation Protocol For Heterogeneous Wireless Sensor Networks," International Journal of Computers, Communication and Control, Romania, Vol. 6, 01, 2011, pp. 113-124.

[6] W. Heinzelman, "Application-Specific Protocol Architectures for Wireless Networks," Ph.D. Thesis, Department of Electrical Engineering and Computer Science, MIT, Cambridge, MA, June 2000.

[7] B. Krishnamachari, D. Estrin, and S.B. Wicker, "The impact of data aggregation in wireless sensor networks," ICDCS Workshop on Distributed Event-based Systems (DEBS), 2002.

[8] E. Fasolo, M. Rossi, J. Widmer, and M. Zorzi, "Innetwork aggregation techniques for wireless sensor networks: a survey," IEEE Wireless Communications, vol. 14, no. 2, pp. 70-87, 2007.

[9] M. Watfa, W. Daher, and H.A.Azaar, "A sensor Network Data Aggregation Technique." International Journal of Computer Theory and Engineering (IJCTE) (Academy Publishers) Volume: 3, No: 1, Page(s): 19-26, 2009.

[10] R. Rajagopalan and P.K. Varshney, "Data-Aggregation Techniques in Sensor Networks: A Survey," IEEE Communication Surveys and Tutorials, Vol. 8 (4), pp. 48-63, December 2006.

[11] K. Maraiya, K. Kant,and N. Gupta, "Wireless Sensor Network: A Review on Data Aggregation," International Journal of Scientific \& Engineering Research Volume 2, Issue 4, April -2011, ISSN 2229-5518.

[12] K. Maraiya, K. Kant, and N. Gupta, "Architectural Based Data Aggregation Techniques in Wireless Sensor Network: A Comparative Study," International Journal on Computer Science and Engineering (IJCSE), Vol. 3 No. 3 Mar 2011 , ISSN : 0975-3397.

[13] R. Jain, "The Art of Computer Systems Performance Analysis: Techniques for Experimental Design, Measurement, Simulation, and Modeling," John Wiley \& Sons, New York, 1991.

[14] G. Bolch, S. Greiner, H. de Meer, and K. S. Trivedi, "Queueing Network and Markov Chains," John Wiley \& Sons, Book, 1998.

[15] F. Baskett, K. M. Chandy, R. R. Muntz, and F. G. Palacios, "Open, closed and mixed networks of queues with different classes of customers," Journal of the ACM 22(2), pp. 248-260, 1975. 